\title{
Análisis de la intención de emprendimiento en estudiantes universitarios de la amazonia ecuatoriana
}

\section{Analysis of the intention of entrepreneurship in university students of the ecuadorian amazon}

Luis Oswaldo Manosalvas Vaca

Viviana Catalina Solís Morejón

Carlos Anibal Manosalvas Vaca

Universidad Estatal Amazónica, Ecuador

Autor para correspondencia: 1manosalvas@uea.edu.ec; vivikatty24@gmail.com;

cmanosalvas@uea.edu.ec

Fecha de recepción: 20 de julio de 2018 - Fecha de aceptación: 30 de agosto de 2018

Resumen: El estudio del espíritu empresarial se ha convertido en un campo de investigación dinámico y diferenciado, en donde las intenciones de emprendimiento se han considerado como uno de los antecedentes de acción de emprendimiento real. Las intenciones se utilizan para describir una predicción individual que permite desarrollar un comportamiento fundamental en el desarrollo social, económico y humano de los países y regiones. La presente investigación analiza la intención de emprendimiento de estudiantes universitarios de la Amazonía ecuatoriana, considerando, que la región Amazónica tiene un potencial económico en desarrollo específicamente por su actividad turística. Para realizar este estudio se utilizó una metodología de investigación cuantitativa, de carácter descriptivo y con un horizonte transversal, se aplicaron técnicas estadísticas descriptivas, inferenciales y multivariantes por medio de un análisis factorial confirmatoria y una relación de ecuaciones estructurales MES. Los resultados demostraron que las dimensiones Norma Subjetiva y Autoeficacia Emprendedora se relacionan de manera significativa con la Intencion de Emprendimiento, sin embargo, la Actitud no tiene una influencia significativa sobre la Intención Emprendedora, estos resultados permiten contribuir en el desarrollo de las habilidades empresariales en los futuros emprendedores de la región Amazónica.

Palabras Claves: intención de emprendimiento; autoeficacia emprendedora; actitud; norma subjetiva

Abstract: Entrepreneurship has become a field of dynamic and differentiated research, where the entrepreneurship intentions have been considered as one of the antecedents of real entrepreneurship. The intentions are used to describe an individual prediction that allows to develop a fundamental behavior in the social, economic and human of the countries and regions. This research analyzes the entrepreneurial intention in university students from the Ecuadorian Amazon, considering that the Amazon region has an economic potential in development specifically tourism activity. In order to carry out this study, a quantitative research methodology was used, with descriptive and transversal analysis. Descriptive, inferential and multivariate statistical techniques were applied through a confirmatory factorial analysis and a relation structural equations SEM. The results showed that the subjective norm and entrepreneurial self- 
efficacy dimensions are significantly related to the Entrepreneurship Intention, however, the Attitude does not have a significant influence on the Entrepreneurial Intention, these results allow to contribute in the development of business skills in the future entrepreneurs of the Amazon region.

Key Words: entrepreneurial intention; entrepreneurial self-efficacy; attitude; subjective standard

\section{Introducción}

El emprendimiento se define como la identificación y explotación de oportunidades comerciales dentro de un contexto de oportunidades individuales, siendo el espíritu empresarial importante para la creación de empleos, el avance económico y social de las naciones y las innovaciones (Van Praag \& Versloot 2007). Además, las grandes empresas están intentando ser más emprendedoras en sus enfoques, debido a esta importancia, el espíritu empresarial se ha convertido en un campo de investigación dinámico y diferenciado (Frese \& Gielnik, 2014). El emprendimiento es una de las áreas de investigación de mayor expansión en las últimas décadas por reconocer que la creación de nuevas empresas se relaciona con el crecimiento económico de los países, la generación de empleo y desarrollo de los mercados (Bahadori, 2012). Varios estudios han considerado las intenciones de emprendimiento como uno de los antecedentes cruciales de acciones de emprendimiento reales (Krueger, 2009). Las intenciones se han utilizado para describir una predicción individual para desarrollar un comportamiento (Ajzen, 1991). Según Ajzen (1991) en la teoría del comportamiento planificado las intenciones se explican por: a) las actitudes del sujeto hacia la conducta; b) las normas subjetivas; y c) la percepción del sujeto de control de la conducta. El estudio de la intención de emprendimiento se puede desarrollar ampliando el contexto del significado de emprendimiento, el mismo que involucra algunos aspectos relevantes cuando el emprendedor inicia y organiza una empresa de negocio, identifica una oportunidad y desarrolla las herramientas, habilidades gestionando el riesgo, supervisa sus esfuerzos para atraer clientes y generar ingresos, por lo menos por un período inicial, asumiendo el riesgo de aventurarse, generalmente por invertir su propio capital y reputación, abandonando un ingreso garantizado (Pozen, 2008). El presente estudio analiza la Intención de Emprendimiento de estudiantes universitarios de la Amazonía ecuatoriana, considerando que la región Amazónica tiene un potencial desarrollo turístico, y los futuros profesionales mediante sus actividades e intenciones empresariales pueden ayudar al crecimiento económico del sector.

\section{Fundamento teórico}

El desarrollo económico se establece por los diferentes emprendimientos que existen en una región, su generación ayuda a la competitividad del mercado. Se ha determinado, que el turismo es una actividad dinámica, generadora de recursos económicos en los diferentes países. El Ecuador, posee una riqueza turística reconocida a nivel mundial lo cual ha permitido al turista extranjero y nacional conocer la diversidad que posee el país, en especial por poseer una región Amazónica que todavía está en vía de desarrollo turístico (Caiza \& Molina, 2012). En la actualidad, el turismo es una fuente que incentiva a la inversión, es por ello que se ha generado un incremento en la necesidad de generar emprendimientos en el sector turístico, los cuales pueden ser afectados por diferentes factores de tipo conductual, financiero, ambiental y político, por lo que es importante considerar las decisiones que se tomen al respecto (Lado, Vivel, Otero, \& Neira, 2015). En el año 2016, según el Global Entrepreneurship Monitor (GEM) indicó que el Ecuador es el país con más actividad emprendedora de la región latinoamericana, situándose 
entre los primeros lugares de los países que se realiza el análisis, sin embargo, en cuanto a la creación de empleo, el establecimiento de los negocios ecuatorianos es muy bajo, tanto así que en los próximos años no se espera generar plazas de trabajo, lo cual limitaría la atracción de clientes internacionales, comparándolos con Colombia y Chile que poseen una alta demanda. De la misma manera, se ha determinado que el Ecuador tiene un alto porcentaje en la Intención de Emprender comparado con otros países de la región (Lasio, Caicedo, Ordeñana, \& Samaniego, 2016).

Uno de los modelos más reconocido que analiza la Intención de Emprender se basa en la Teoría de Ajzen, también conocida como la Teoría del Comportamiento Planificado en donde las intenciones se explican por: (a) Las actitudes del sujeto hacia la conducta; que se refiere al grado en que la persona realiza una evaluación positiva o negativa de un comportamiento. Así, una actitud favorable estará asociada a que la intención de llevar a cabo una conducta sea mayor. (b) Las normas subjetivas que se indican como un razonamiento preceptivo que pone de manifiesto la presión social percibida por la persona que va a realizar la acción dirigida hacia la ejecución o no de dicha acción; y (c) La percepción del sujeto de control de la conducta, determinada por la autoeficacia emprendedora que se conceptualiza como la confianza de la persona en sus propias habilidades para llevar a cabo eficazmente las acciones y tareas específicas implicadas en la creación y desarrollo de una nueva empresa. Davidsson (1995) sugirió que las Intenciones de Emprendimiento pueden ser influenciados por: La convicción, que se define por actitudes generales (cambio, competir, dinero, logro y la autonomía), las actitudes de dominio (rentabilidad, contribución hacia la sociedad y el conocimiento práctico o habilidad); la convicción, a su vez, se relaciona con variables personales como la edad, el género, la educación, la experiencia vicaria y la experiencia del cambio radical. Se reportan diversos estudios que han utilizado estos modelos de Intención Emprendedora, sin embargo, se necesita más investigación porque puede existir otros factores relevantes que pueden influir en la Intención de Emprendimiento, en especial en lugares y regiones que todavía no se encuentran desarrolladas económicamente. Sin embargo, existen nuevas alternativas de estudio en esta área, la propuesta de nuevas ideas y perspectivas se basa en las categorías de investigación, o enfoques conceptuales: (a) La primera categoría abarca trabajos que estudian el modelo de Intención Emprendedora, ya sea profundizando el conocimiento de algunos matices teóricos, o el análisis de cuestiones metodológicas; (b) La segunda categoría se analiza el papel de las variables a nivel personal en la configuración de las Intenciones de Emprendimiento; (c) Un tercer grupo de trabajos se refiere a la relación que existe entre la educación empresarial y la intención emprendedora de sus participantes; d) El rol del contexto y las instituciones juegan un papel relevante en la configuración de las intenciones empresariales lo que constituye la cuarta categoría; e) Por último, se considera el proceso emprendedor y el enlace con la Intención de emprendimiento (Fayolle \& Liñan, 2014).

La educación empresarial busca construir conocimiento y habilidades, y también aumentar la probabilidad de éxito del negocio, por lo que los comportamientos empresariales necesitan ser aprendido, a partir de las primeras etapas de la educación de un niño, a más tardar durante los años de la escuela secundaria (Souitaris, Zerbinati \& Al-Laham, 2007). Las universidades se están convirtiendo en entidades que cooperan y se relacionan con la industria, las actividades empresariales en las universidades, incluyendo la evolución de los planes de estudio y la infraestructura, están aumentando a un ritmo significativo (Katz, 2003). Muchos 
estudios que se han realizado han indicado que la educación empresarial juega un papel importante para cultivar el espíritu empresarial entre los graduados universitarios. El emprendimiento en las instituciones académicas convencionalmente se ha asociado con la educación empresarial y la formación en el contexto de la creación de las pequeñas y nuevas empresas (Carlsson, Acs, Audretsch, \& Braunerhjelm, 2009).

En las universidades ecuatorianas todavía existe un patrón en el cual los estudiantes una vez que finalizan sus estudios, tratan de emplearse y no buscan alternativas para generar un emprendimiento, en Ecuador los que tienen una mayor intención de emprender son los que han culminado su nivel secundario de educación; comparado con estudiantes universitarios y de posgrado, donde la intencionalidad empieza a decrecer (Lasio, Caicedo, Ordeñana, \& Samaniego, 2016). Por lo tanto, se plantea la siguiente pregunta de investigación: ¿Existen intenciones para desarrollar emprendimientos en los estudiantes universitarios de la Amazonía ecuatoriana?, con esta pregunta de investigación se plantearaon las siguientes hipótesis

- H1: La Norma Subjetiva influye positivamente en la intención de emprendimiento

- H2: La Autoeficacia emprendedora influye positivamente en la intención de emprendimiento

- H3: Las Actitudes influyen positivamente en la intención de emprendimiento

\section{Métodos}

La Intención de Emprendimiento se ha convertido en una de las áreas relevantes para entender el fenómeno emprendedor (Liñán \& Chen, 2009). El diseño de la investigación utilizó: (a) un enfoque cuantitativo, con una lógica deductiva que analiza los factores psicológicos propios del emprendedor que anteceden a la Intención de Emprendimiento; (b) tiene un propósito descriptivo que indica y analiza detalles específicos de los factores psicológicos que anteceden a la Intención de Emprendimiento y sus relaciones con el entorno social; (c) sigue un horizonte transversal que integra un análisis de las variables propuestas en el marco conceptual en un tiempo determinado; (d) la investigación utiliza la encuesta como método de medición cuantitativo para analizar los factores conductuales que influyen en las Intenciones de Emprendimiento de los estudiantes universitarios, los instrumentos utilizados son válidos y confiables por autores consultados en el área de la relación de características psicológicas y emprendimiento. El diseño de una investigación cuantitativa fue el más adecuado para este trabajo, (a) el planteamiento del estudio fue desarrollado a partir de un marco conceptual que explica los factores psicológicos conductuales en la Intención de Emprendimiento. (b) existe el respaldo de literatura científica relevante sobre el tema que ha seguido un paradigma cuantitativo (Liñan \& Chen, 2009); (c) el problema de investigación tiene un carácter descriptivo orientado a desarrollar explicaciones específicas; (d) el estudio involucra un propósito específico y medible en un lapso de tiempo a partir de datos observables y cuantificables; (e) Se recogen datos numéricos usando encuestas como instrumentos de medición dirigidos hacia estudiantes universitarios de la Amazonía ecuatoriana; (f ) se requiere un análisis estadístico descriptivo y multivariante para el análisis e interpretación de datos con la finalidad de realizar análisis descriptivos e inferenciales, relacionar variables, evaluar resultados con estudios anteriores; $(\mathrm{g})$ Los datos obtenidos son objetivos y se reportan mediante la presentación de artículo científico. 
La población de estudio considera ciertos atributos que podrían influir en el momento de crear un emprendimiento: como la edad, el nivel de conocimiento, la experiencia laboral, y según modelos de referencia familiar. La naturaleza de la investigación está dirigida a la obtención de datos primarios de una población distribuida en universidades de la amazonía ecuatoriana como son la Universidad Estatal Amazónica y Escuela Superior Politécnica del Chimborazo, la muestra seleccionada fueron estudiantes de los últimos semestres de las carreras de Ingeniería en Turismo y también de Contabilidad y Auditoría, respectivamente, con un número de 326 estudiantes, se aplicó un muestreo estratificado. Se analizó el modelo de Intenciones empresariales propuesto por el Dr. Francisco Liñán PhD en el año 2009, el cual está basado en la Teoría de Comportamiento Planificado desarrollado por Ajzen en el año1991.

\section{Resultados}

\section{Datos demográficos}

Tabla 1. Género y Edad de la muestra seleccionada

\begin{tabular}{cccc}
\hline GENERO & $\%$ & EDAD & $\%$ \\
\hline Hombre & 45 & $18-24$ años & 65 \\
Mujer & 55 & $18-22$ años & 72
\end{tabular}

El mayor porcentaje corresponde a al género femenino en comparación al masculino, además, la edad fluctúa entre los 18 a 24 años en ambos géneros.

Tabla 2. Experiencia laboral de la muestra seleccionada

\begin{tabular}{cc}
\hline EXPERIENCIA & $\%$ \\
AUTOEMPLEADO & \\
\hline Relación de dependencia & 82 \\
Negocios propios & 18 \\
\hline
\end{tabular}

El mayor porcentaje ha indicado que no tiene experiencia laboral, sin embargo, existe un $42 \%$ que tiene experiencia laboral entre 1 a más de 2 años.

\begin{tabular}{|c|c|}
\hline $\begin{array}{l}\text { EXPERIENCIA } \\
\text { LABORAL }\end{array}$ & $\%$ \\
\hline 0 años & 58 \\
\hline 1 año & 18 \\
\hline 2 años & 8 \\
\hline Más de 2 años & 16 \\
\hline
\end{tabular}

Los estudiantes que indican que han trabajado la mayor cantidad lo ha realizado en relación de de dependencia y apenas un $18 \%$ ha incursionado en negocios propios.

Tabla 4. Nivel de Educación de padres

\begin{tabular}{ccc}
\hline NIVEL EDUCACION PADRES & PADRE & MADRE \\
\cline { 2 - 3 } & $\%$ & $\%$ \\
Escuela & 42 & 43 \\
Bachillerato & 34 & 35 \\
Universidad/Técnico Profesional & 24 & 22 \\
\hline
\end{tabular}


Los padres de la muestra seleccionada en su mayoría han llegado a un nivel de escuela, el otro porcentaje mayoritario ha culminado el bachillerato, sin embargo, existe un bajo porcentaje ha cursado estudios superiores en universidades y son técnicos profesionales.

\section{Análisis factorial confirmatorio modelo intenciones empresariales}

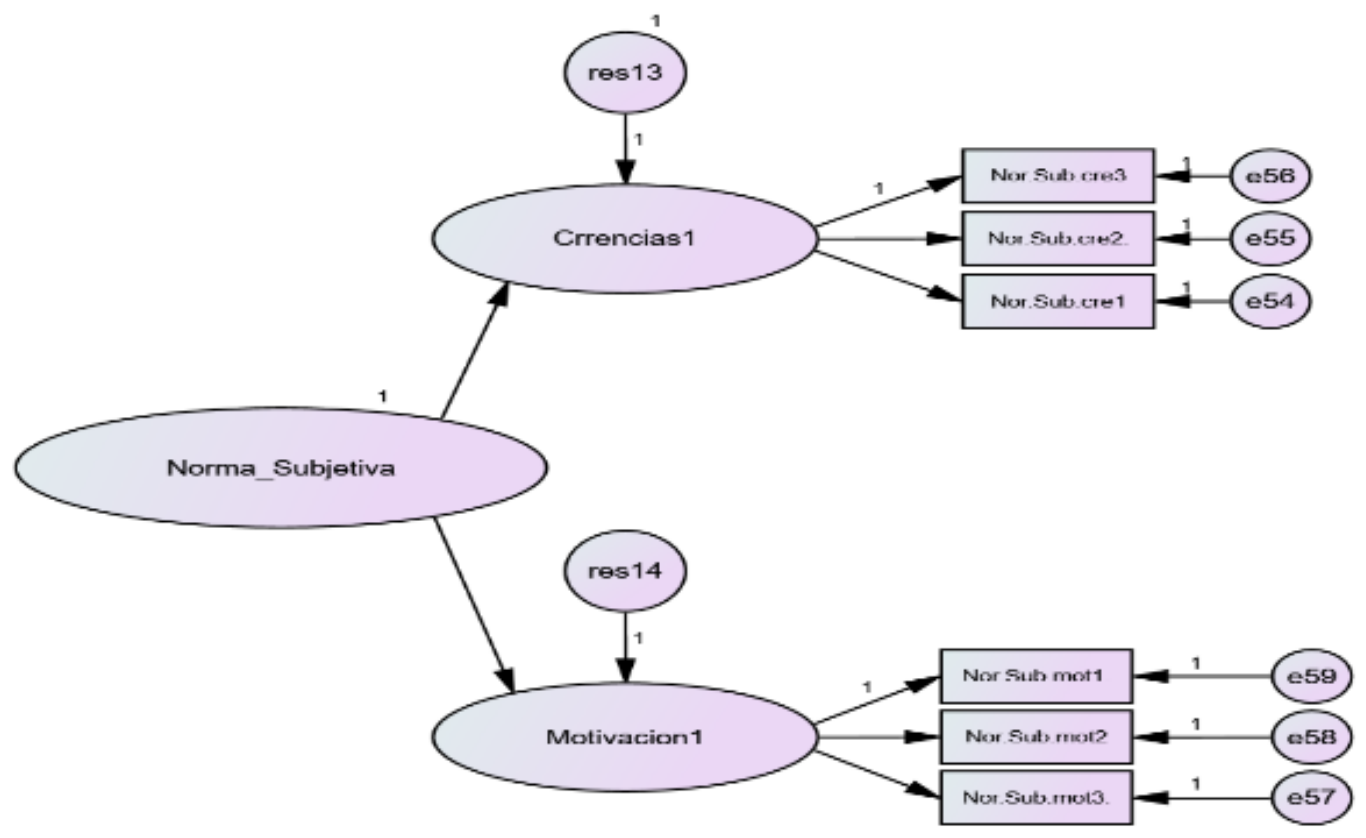

Figura1. Modelo de Norma Subjetiva

Tabla 5. Índices de Bondad de Ajuste Modelo Norma Subjetiva

\begin{tabular}{ccc}
\hline Índice & Valor & Nivel de Aceptación \\
\hline Chi-cuadrado & 11.925 & $\mathrm{P}<0.2$ \\
GI & 6 & \\
CFI & 0.953 & $\geq 0.95$ \\
RMSEA & 0.059 & $\leq 0.06$ \\
GFI & 0.941 & $>0.90$ \\
AGFI & 0.890 & $>0.80$ \\
CMIN/DF & 2.948 & $<5$
\end{tabular}

El modelo de Norma Subjetiva indica un buen ajuste ya que todos los índices se encuentran dentro de los niveles aceptables.

Tabla 6. Validez Convergente Norma Subjetiva

\begin{tabular}{lcllll}
\hline & & & Estimados & S.E. & P $\leq \mathbf{0 , 0 0 5}$ \\
\hline Creencias1 & $<--$ & Norma_Subj & 0,106 & 0,187 & $* * *$ \\
Motivacion1 & $<--$ & Norma_Subj & 1,428 & 1,563 & $* * *$ \\
Nor.Sub.cre3.compañeros_colegas & $<--$ & Creencias1 & 1 & & \\
Nor.Sub.cre2.amigos_íntimos & $<---$ & Creencias1 & 1,324 & 0,123 & $* * *$ \\
Nor.Sub.cre1.familia_directa & $<---$ & Creencias1 & 0,386 & 0,087 & $* * *$ \\
\hline
\end{tabular}




\begin{tabular}{lllllll}
\hline Nor.Sub.mot1.familia_padres_hermanos & $<---$ & Motivacion1 & 1 & & & \\
Nor.Sub.mot2.amigos_cercanos & $<---$ & Motivacion1 & 4,206 & 1,342 & $* * *$ \\
Nor.Sub.mot3.compañeros & $<---$ & Motivacion1 & 4,104 & 1,229 & $* * *$ \\
\hline
\end{tabular}

El análisis factorial confirmatorio indica que la validez convergente del modelo Norma subjetiva es válido al demostrar una significancia adecuada entre los ítems y los constructos.

\begin{tabular}{ccc} 
Tabla 7. Índices de Bondad de Ajuste Modelo Act \\
\cline { 2 - 3 } Índice & Valor & Nivel de Aceptación \\
& & \\
\hline Chi-cuadrado & 187.567 & $\mathrm{P}<0.00$ \\
Gl & 45 & \\
CFI & 0.902 & $\geq 0.95$ \\
RMSEA & 0.062 & $\leq 0.06$ \\
GFI & 0.932 & $>0.90$ \\
AGFI & 0.820 & $>0.80$ \\
CMIN/DF & 4.564 & $<5$ \\
\hline
\end{tabular}

El modelo Actitudes indica un buen ajuste ya que todos los índices se encuentran dentro de los niveles aceptables.

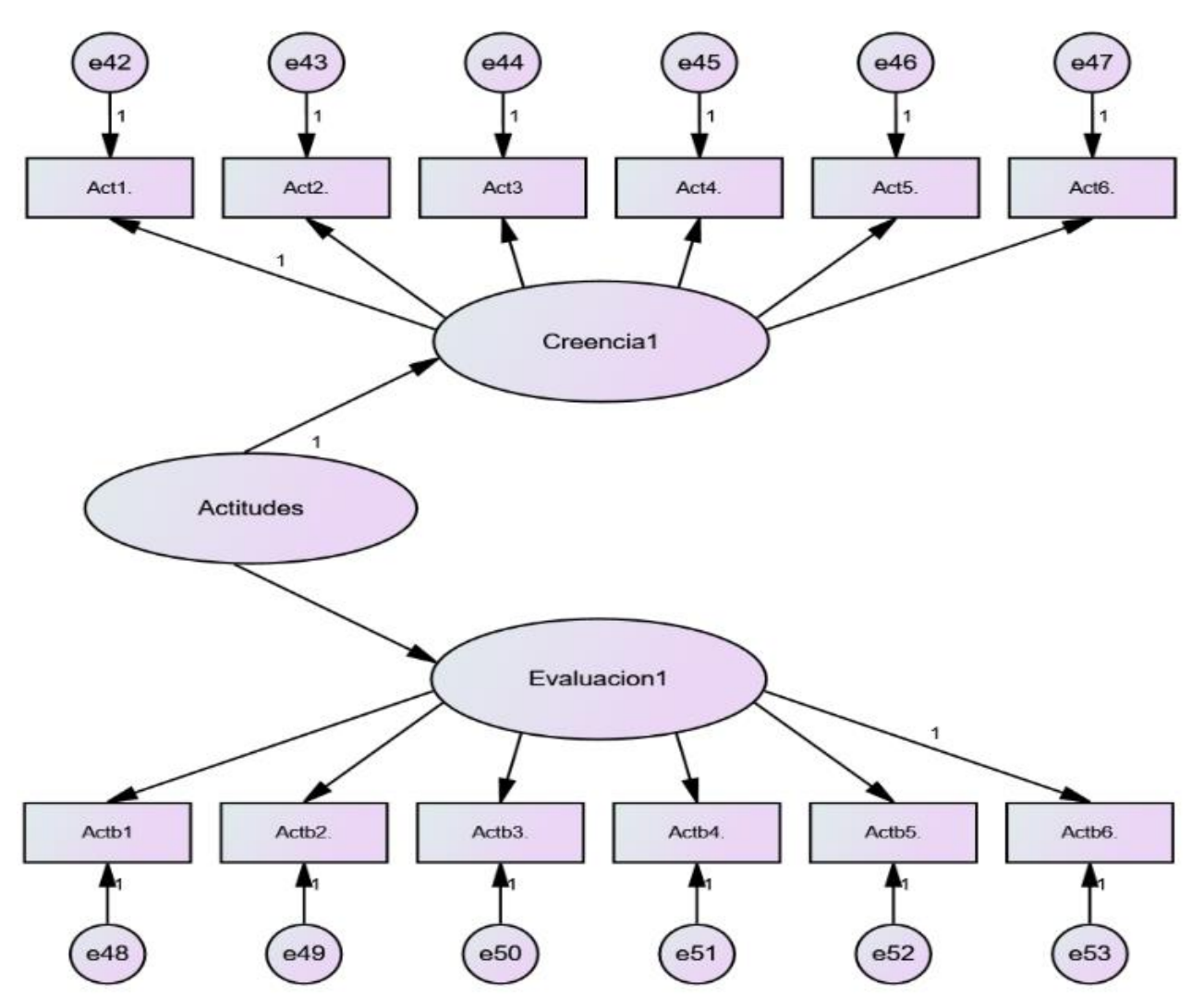

Figura 2. Modelo Actitudes

Tabla 8. Validez Convergente Modelo Actitud 


\begin{tabular}{|c|c|c|c|c|c|}
\hline & & & Estimados & Error Estándar. & $\mathrm{P} \leq \mathbf{0 , 0 0 5}$ \\
\hline Creencia1 & $<--$ & Actitudes & 0,172 & 0,067 & $* * *$ \\
\hline Evaluacion1 & $<--$ & Actitudes & 2,788 & 0,303 & $* * *$ \\
\hline Act1.nuevos_retos & $<---$ & Creencia1 & 1 & & \\
\hline Act2.crear_empleo & $<---$ & Creencial & 0,945 & 0,093 & $* * *$ \\
\hline Act3.creativo_innovador & $<---$ & Creencia1 & 0,901 & 0,09 & $* * *$ \\
\hline Act4.altos_ingresos_económicos & $<---$ & Creencia1 & 0,856 & 0,99 & $* * *$ \\
\hline Act5.riesgos_calculados & $<---$ & Creencial & 0,867 & 0,152 & $* * *$ \\
\hline Act6.propio_jefe & $<---$ & Creencia1 & 0,798 & 0,087 & $* * *$ \\
\hline Actb6.independiente_trabajo & $<---$ & Evaluacion1 & 1 & & \\
\hline Actb5.asumir_riesgos & $<--$ & Evaluacion 1 & 1,348 & 0,163 & $* * *$ \\
\hline Actb4.tener_mucho_dinero & $<---$ & Evaluacion1 & 1,455 & 0,176 & $* * *$ \\
\hline Actb3.creatividad_innovación & $<--$ & Evaluacion1 & 1,657 & 0,14 & $* * *$ \\
\hline Actb2.crear_empleos & $<--$ & Evaluacion1 & 1,45 & 0,143 & $* * *$ \\
\hline Actb1.enfrentarse nuevos retos & $<--$ & Evaluacion1 & 1,281 & 0,134 & $* * *$ \\
\hline
\end{tabular}

$\mathrm{El}$ análisis factorial confirmatorio indica que la validez convergente del modelo Actitudes es válido al demostrar una significancia adecuada entre los ítems y los constructos.

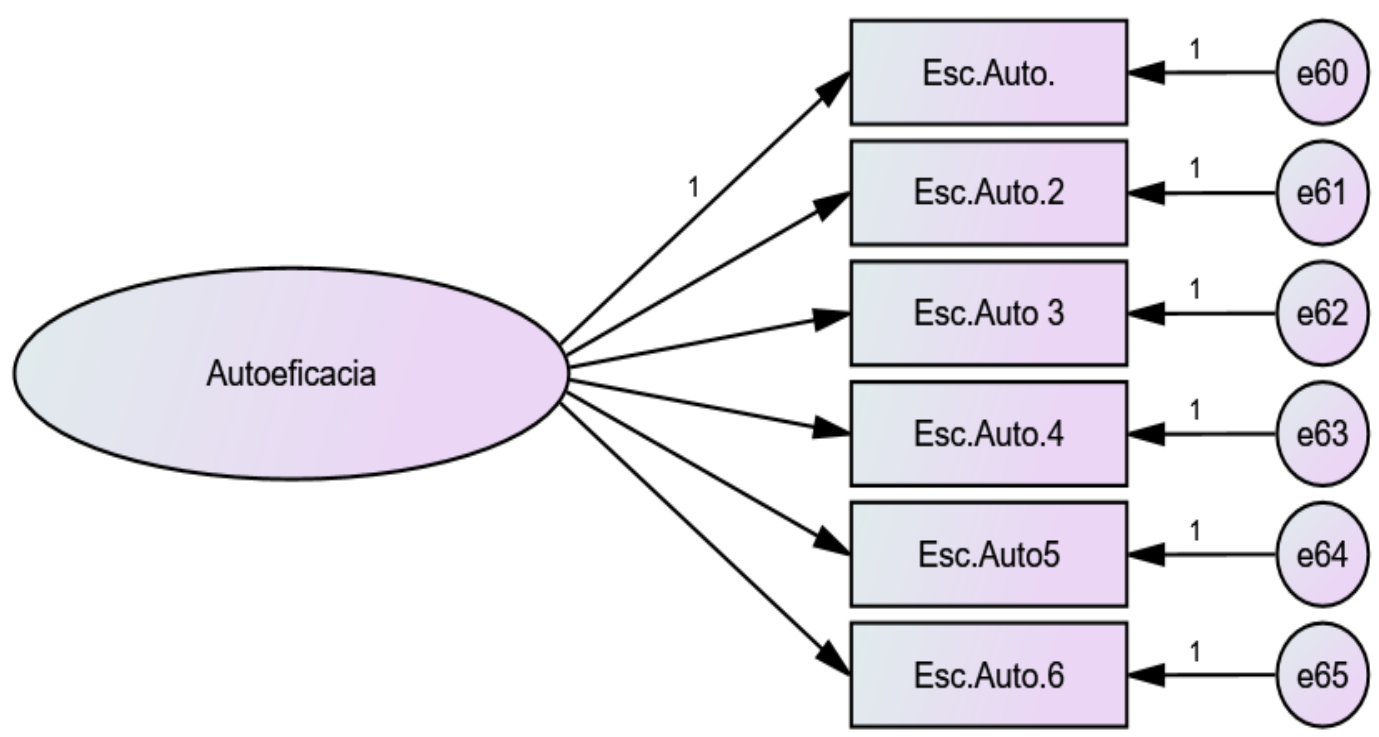

Figura 3. Modelo Autoeficacia Emprendedora

Tabla 9. Índices de Bondad de Ajuste Modelo Autoeficacia Emprendedora 


\begin{tabular}{ccc}
\hline Índice & Valor & Nivel de Aceptación \\
\hline Chi-cuadrado & 30.856 & $\mathrm{P}<0.02$ \\
Gl & 4 & \\
CFI & 0.937 & $\geq 0.95$ \\
RMSEA & 0.161 & $\leq 0.06$ \\
GFI & 0.987 & $>0.90$ \\
AGFI & 0.889 & $>0.80$ \\
CMIN/DF & 4.560 & $<5$ \\
\hline
\end{tabular}

El modelo Autoeficacia Emprendedora indica un buen ajuste ya que todos los índices se encuentran dentro de los niveles aceptables.

Tabla 10. Validez Convergente modelo Autoeficacia Emprendedora

\begin{tabular}{|c|c|c|c|c|c|}
\hline & & & Estimate & S.E. & $P \leq 0,005$ \\
\hline Esc.Auto.idea_negocio_estrategia & $\begin{array}{c}<-- \\
-\end{array}$ & Autoeficacia1 & 1 & & \\
\hline Esc.Auto.control_nueva_empresa & $\begin{array}{c}<- \\
-\end{array}$ & Autoeficacial & 1,013 & 0,102 & $* * *$ \\
\hline Esc.Auto.negociar & $\begin{array}{c}<-- \\
-\end{array}$ & Autoeficacial & 1,037 & 0,105 & $* * *$ \\
\hline Esc.Auto.oportunidades_mercado & $\begin{array}{c}<-- \\
-\end{array}$ & Autoeficacia1 & 0,998 & 0,101 & $* * *$ \\
\hline Esc.Auto.relación_personas_claves & $\begin{array}{l}<- \\
-\end{array}$ & Autoeficacial & 0,997 & 0,097 & $* * *$ \\
\hline Esc.Auto.crear_nueva_empresa & $\begin{array}{c}<-- \\
- \\
\end{array}$ & Autoeficacial & 0,941 & 0,096 & $* * *$ \\
\hline
\end{tabular}

El modelo Autoeficacia Emprendedora es válido al demostrar una significancia adecuada entre los ítems y los constructos.

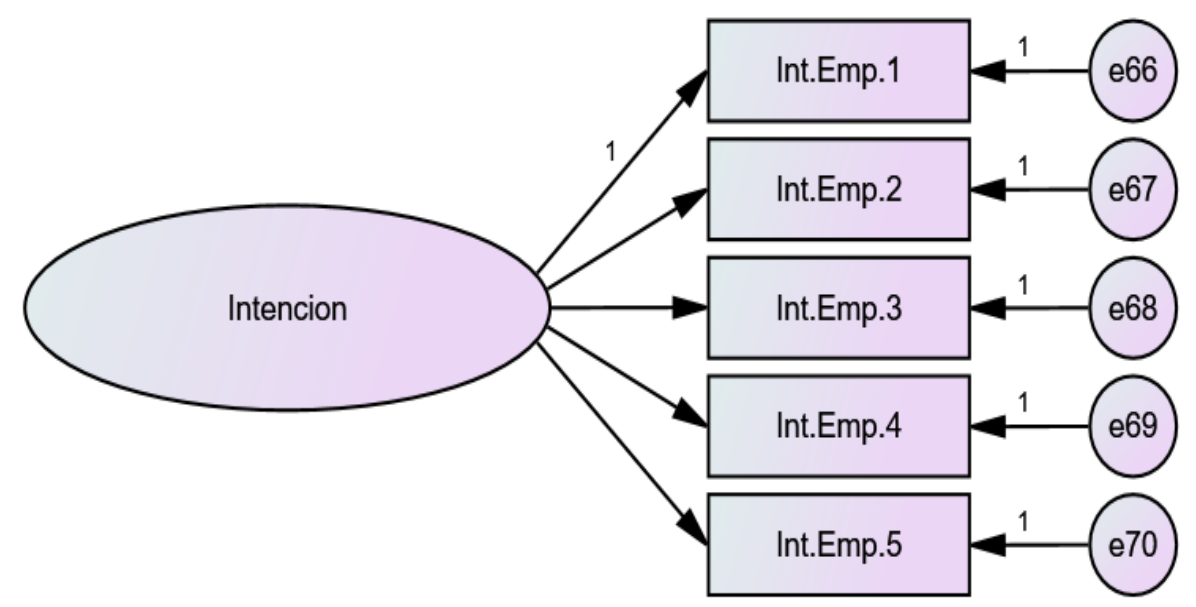

Figura 4. Modelo Intención de Emprendimiento

Tabla 11. Índices de Bondad de Ajuste Modelo Intención de Emprendimiento 


\begin{tabular}{ccc}
\hline Índice & Valor & Nivel de Aceptación \\
\hline Chi-cuadrado & 30.856 & $\mathrm{P}<0.02$ \\
Gl & 4 & \\
CFI & 0.937 & $\geq 0.95$ \\
RMSEA & 0.161 & $\leq 0.06$ \\
GFI & 0.987 & $>0.90$ \\
AGFI & 0.889 & $>0.80$ \\
CMIN/DF & 4.560 & $<5$ \\
\hline
\end{tabular}

El modelo Intención de Emprendimiento indica un buen ajuste ya que todos los índices se encuentran dentro de los niveles aceptables.

Tabla 12. Validez Convergente modelo Intención de Emprendimiento

\begin{tabular}{ccccccc}
\hline & & & Estimate & S.E. & P \\
\hline Int.Emp.crear_empresa_algún_día & $<---$ & Intencion1 & 1 & & \\
Int.Emp.esfuerzo_empresario & $<---$ & Intencion1 & 0,871 & 0,202 & $* * *$ \\
Int.Emp.dudas_creación_empresa & $<---$ & Intencion1 & 0,315 & 0,123 & $* * *$ \\
Int.Emp.empresa_futuro & $<---$ & Intencion1 & 1,068 & 0,234 & $* * *$ \\
Int.Emp.objetivo_ser_empresario & $<---$ & Intencion1 & 1,234 & 0,143 & $* * *$ \\
\hline
\end{tabular}

El análisis convergente del modelo Intención de Empendimiento es válido al demostrar una significancia adecuada entre los ítems y los constructos.

Modelo ecuaciones estructurales intenciones empresariales

Tabla 13. Índices de regresión variables latentes

\begin{tabular}{cc}
\hline Variables & Nivel de Significancia \\
\hline Actitudes - Intención de Emprendimiento & 0,226 \\
Norma Subjetiva - Intención de Emprendimiento & $* * *$ \\
Autoeficacia - Intención de Emprendimiento & $* * *$ \\
\hline
\end{tabular}




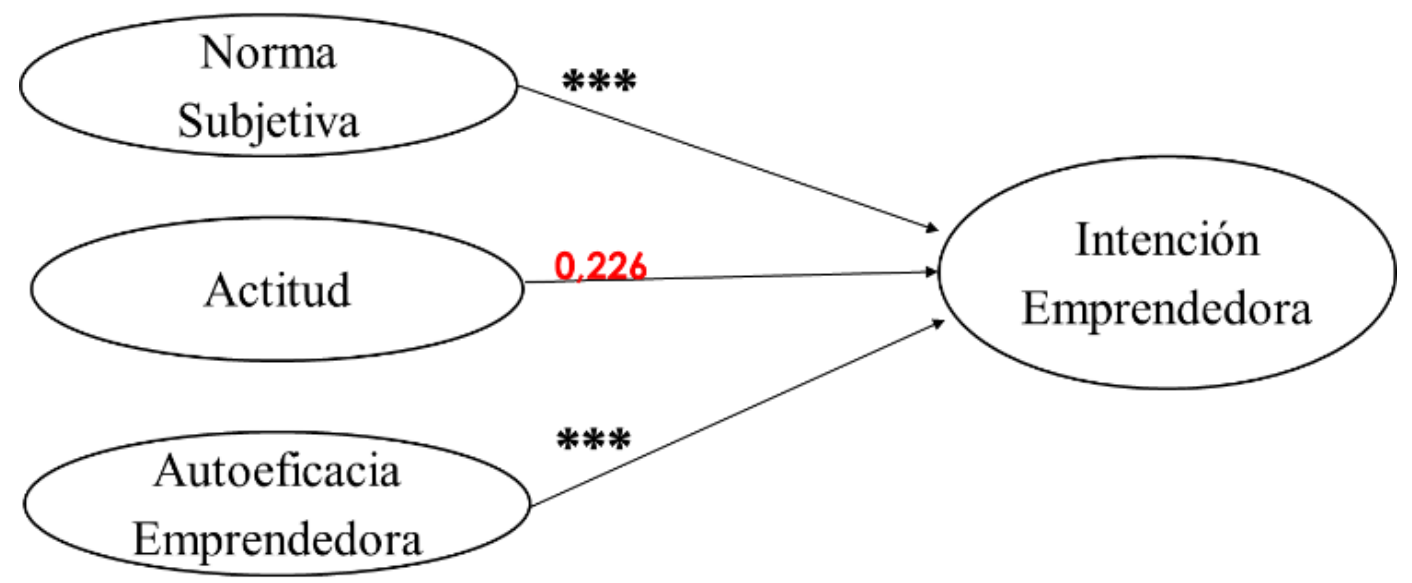

Figura 4. Modelo de Ecuaciones estructurales Intención de Emprendimiento

\section{Discusión}

El estudio demográfico indica que en la muestra analizada existe un mayor porcentaje de mujeres en comparación con los hombres, la edad predominante de los estudiantes va desde los 18 a 24 años en ambos géneros, el mayor porcentaje de la muestra seleccionada no tiene experiencia en el ámbito laboral, sin embargo, se puede evidenciar que los estudiantes que han trabajado lo han hecho en su mayoría en relación de dependencia, y apenas un bajo porcentaje a logrado generar un trabajo como auto empleado en un negocio propio. El nivel más alto de estudio alcanzado por los padres de los encuestados es la primaria, seguido por el bachillerato, por lo tanto, se puede evidenciar que no existe una influencia en lo que se refiere a educación empresarial por parte de los progenitores. La validez convergente evalúa si los constructos con sus preguntas miden lo que tienen que medir de manera significativa y la validez discriminante indica que cada constructo debe medir en forma diferente (Chion \& Vincent, 2016). Todos los estimados de cada ítem hacía los subconstructos son significativos ( $p$-valor $<0.05$ ), por lo tanto, se indica que existe validez convergente (Anderson \& Gerbing, 1988). La validez discriminante, que es la verificación de que no exista correlación entre los constructos, señaló que todas las diferencias son significativas, por lo tanto, se puede evidenciar que los instrumentos de medición tienen dimensiones que miden de forma diferente (Chion \& Vincent, 2016). El Análisis Factorial Confirmatorio ha demostrado que el instrumento utilizado, al igual que los modelos de los constructos analizados son significativos y válidos en su aplicación, de la misma manera, el modelo de ecuaciones estructurales SEM respecto a las relaciones de las variables evidencian que los constructos Norma Subjetiva, Autoeficacia Emprendedora se relacionan de manera significativa y positiva con la Intención Emprendedora, sin embargo, el constructo Actitud no tiene una relación significativa con la Intención Emprendedora.

\section{Conclusión}

Con los resultados de la investigación se puede concluir, que los estudiantes de la región amazónica tienen una alta intención de realizar emprendimientos, considerando el entorno social familiar y social que lo rodea, como así lo indica la Norma Subjetiva, de la misma manera, se evidencia que existe una alta creencia en sus propias habilidades emprendedoras (Autoeficacia Emprendedora), lo cual influye en el momento de desarrollar la intención de emprender, sin embargo, la Actitud a desarrollar emprendimientos no tiene una influencia significativa sobre la 
Intención de Emprendimiento, considerando que esta variable se trata de una capacidad propia de los seres humanos con la que enfrentan el mundo y las circunstancias que se les podrían presentar en la vida real, las universidades tienen que potencializar las habilidades emprendedoras mediante el desarrollo de capacidades blandas y simulaciones de emprendimiento en incubadoras empresariales estudiantiles. Se recomienda seguir la investigación sobre la Actitud en la Intención de Emprendimiento en otros contextos laborales y regionales para determinar los posibles factores que se encuentran involucrados con este comportamiento emprendedor.

\section{Bibliografía}

Ajzen, I. (1991). The Theory of Planned Behaviour. Organizational Behaviour and Human Decision Processes, 50(2), 179-211.

Anderson, J; Gerbing, D. (1988). Structural Equation Modeling in Practice: A Review and Recommended Two-Step Approach. Psychological Bulletin, 103, 411-423.

Bahadori, M. (2012). The Effect of Emotional Intelligence on Entrepreneurial Behavior: A Case Study in a Medical Science University. Asian Journal of Business Management, 4(1), 8185 .

Caiza, R., \& Molina, E. (2012). Análisis Histórico de la Evolución del turismo en el Territorio Ecuatoriano. Ricit, 2, 8-24.

Carlsson, B., Acs, Z. J., Audretsch, D., \& Pontus Braunerhjelm. (2009). Knowledge Creation, Entrepreneurship, and Economic Growth: A Historical Review. Industrial and Corporate Change, 18(6), 1193-1229

Chion, S., \& Vincent, Ch. (2016). Analítica de datos para la modelación estructural. Lima, Perú: Pearson.

Davidsson, P. (1995). Determinants of Entrepreneurial Intentions. Jonkoping International Business School. Recuperado de http://eprints.qut.edu.au/2076/1/RENT_IX.pdf

Fayolle, A., \& Liñán, F. (2014). The Future of Research on Entrepreneurial Intentions. Journal of Business Research, 67(5), 663-666.

Frese, M., \& Gielnik, M. M. (2014). The psychology of entrepreneurship. Annual Review of Organizational Psychology and Organizational Behavior, 1(1).

Katz, J. A. (2003). The Chronology and Intellectual Trajectory of American Entrepreneurship Education 1876-1999. Journal of Business Venturing, 18(2), 283-300.

Krueger, N. F. (2009). Entrepreneurial Intentions are Dead: Long Live Entrepreneurial Intentions. In A. L. Carsrud, \& M. Brännback (Eds.), Understanding the Entrepreneurial mind (pp. 51-72). New York, NY: Springer. 
Lado, R; Vivel, M; Otero, L., \& Neira, I,. (2015). Barreras al emprendimiento hotelero: un análisis sectorial. Red de Revistas Científicas de América Latina y el Caribe, España y Portugal, 1, 86-92.

Lasio, Caicedo, Ordeñana, \& Izquierdo (2016). Global Entrepreneurship Monitor (Ed).Offset Abad (pp.27). Quito, Ecuador. Recuperado de file:///G:/reportegem20161498597645.pdf

Liñán, F., \& Chen, Y. (2009). Development and Cross-Cultural Application of a Specific Instrument to Measure Entrepreneurial Intentions. Entrepreneurship: Theory \& Practice, 33(3), 593-617.

Pozen, D. E. (2008). We are all Entrepreneurs Now. Wake Forest Law Review, 43(1), 283- 340.

Souitaris, V., Zerbinati, S., \& Al-Laham, A. (2007). Do Entrepreneurship Programmes Raise Entrepreneurial Intention of Science and Engineering Students? The Effect of Learning, Inspiration and Resources. Journal of Business Venturing, 22(4), 566-591.

Van Praag C.M. \& Versloot P.H. (2007). What is the value of entrepreneurship? A review of recent research. Small Bus. Econ. 29(4), 351-382 